\title{
A new aspect of generalized integral operator and an estimation in a generalized function theory
}

\author{
Shrideh Al-Omari ${ }^{*}$ (D), Hassan Almusawa² (1) and Kottakkaran Sooppy Nisar ${ }^{3}$ (D)
}

${ }^{*}$ Correspondence:
s.k.q.alomari@fet.edu.jo;
shridehalomari@bau.edu.jo
${ }^{1}$ Department of Physics and Basic
Sciences, Faculty of Engineering
Technology, Al-Balqa Applied
University, 11134, Amman, Jordan
Full list of author information is
available at the end of the article

available at the end of the article

\begin{abstract}
In this paper we investigate certain integral operator involving Jacobi-Dunkl functions in a class of generalized functions. We utilize convolution products, approximating identities, and several axioms to allocate the desired spaces of generalized functions. The existing theory of the Jacobi-Dunkl integral operator (Ben Salem and Ahmed Salem in Ramanujan J. 12(3):359-378, 2006) is extended and applied to a new addressed set of Boehmians. Various embeddings and characteristics of the extended Jacobi-Dunkl operator are discussed. An inversion formula and certain convergence with respect to $\delta$ and $\Delta$ convergences are also introduced.
\end{abstract}

MSC: Primary 54C40; 14E20; secondary 46E25; $20 \mathrm{C} 20$

Keywords: Difference operator; Differential-difference function; Jacobi-Dunkl function; Integral transform; Boehmian; Differential-difference operator

\section{Introduction and preliminaries}

We start with some background and notations from the Jacobi-Dunkl function theory, supplementing the material in the Introduction. We recapitulate some results related to the harmonic analysis associated with the Jacobi-Dunkl differential-difference operator $\Delta_{\alpha, \beta}$ and the Jacobi-Dunkl kernel function $\Psi_{\lambda}^{\alpha, \beta}$. We denote by $\mathbb{C}, \mathbb{R}$, and $\mathbb{N}$ the sets of complex numbers, real numbers, and positive integers, respectively. For $\alpha, \beta \in \mathbb{R}, \alpha \geq \beta \geq$ $-\frac{1}{2}$, and $\alpha \neq-\frac{1}{2}$, we denote by $\Delta_{\alpha, \beta}$ the Jacobi-Dunkl differential-difference operator defined by [1]

$$
\Delta_{\alpha, \beta} \psi(\zeta)=\dot{\psi}(\zeta)+((2 \alpha+1) \operatorname{coth} \zeta+(2 \beta+1) \tanh \zeta)\left(\frac{\psi(\zeta)-\psi(-\zeta)}{2}\right) .
$$

For $\lambda^{2}=\mu^{2}+\rho^{2}, \lambda \in \mathbb{C}, \zeta \in \mathbb{R}$, and $\rho=\alpha+\beta+1$, we denote by $\Psi_{\lambda}^{\alpha, \beta}$ the Jacobi-Dunkl kernel function [1]

$$
\Psi_{\lambda}^{\alpha, \beta}(\zeta)=\varphi_{\mu}^{\alpha, \beta}(\zeta)+i \frac{\lambda}{2(\alpha+1)} \sinh \zeta \cosh \zeta \varphi_{\mu}^{\alpha+1, \beta+1}(\zeta)
$$

(c) The Author(s) 2021. This article is licensed under a Creative Commons Attribution 4.0 International License, which permits use sharing, adaptation, distribution and reproduction in any medium or format, as long as you give appropriate credit to the original author(s) and the source, provide a link to the Creative Commons licence, and indicate if changes were made. The images or other third party material in this article are included in the article's Creative Commons licence, unless indicated otherwise in a credit line to the material. If material is not included in the article's Creative Commons licence and your intended use is not permitted by statutory regulation or exceeds the permitted use, you will need to obtain permission directly from the copyright holder. To view a copy of this licence, visit http://creativecommons.org/licenses/by/4.0/. 
Then the Jacobi-Dunkl kernel function $\Psi_{\lambda}^{\alpha, \beta}$ is the $C^{\infty}$-solution of the differentialdifference equation

$$
\left.\begin{array}{l}
\Delta_{\alpha, \beta} \Psi_{\lambda}^{\alpha, \beta}=i \lambda \Psi_{\lambda}^{\alpha, \beta}, \quad \lambda \in \mathbb{C}, \\
\Psi_{\lambda}^{\alpha, \beta}(0)=1,
\end{array}\right\}
$$

where $\varphi_{\mu}^{\alpha, \beta}$ is the Jacobi function defined by

$$
\varphi_{\mu}^{\alpha, \beta}(\zeta)=F\left(\frac{\rho+i \mu}{2}, \frac{\rho-i \mu}{2}, \alpha+1,-(\sinh \zeta)^{2}\right)
$$

$F$ being a Gauss hypergeometric function. For $\alpha \geq \beta \geq-\frac{1}{2}$ and $\lambda \in \mathbb{C}$, the function $\Psi_{\lambda}^{\alpha, \beta}$ is an eigenfunction of the differential-difference operator $\Delta_{\alpha, \beta}$ that satisfies the product formula $[1,(2.10)]$

$$
\Psi_{\lambda}^{\alpha, \beta}(\zeta) \Psi_{\lambda}^{\alpha, \beta}(y)=\int_{-\infty}^{\infty} \Psi_{\lambda}^{\alpha, \beta}(u) d \mu_{\zeta, y}^{\alpha, \beta}(u)
$$

where

$$
d \mu_{\zeta, y}^{\alpha, \beta}(u) \begin{cases}K_{\alpha, \beta}(\zeta, y, u) X_{\alpha, \beta}(u) d u, & \zeta y \neq 0, \\ \delta_{x}, & y=0, \\ \delta_{y}, & \zeta=0,\end{cases}
$$

and

$$
\begin{aligned}
K_{\alpha, \beta}(\zeta, y, u)= & M_{\alpha, \beta}(\sinh |\zeta| \sinh |y| \sinh |u|)^{-2 \alpha} 1_{I_{\zeta, y}}(u) \\
& \times \int_{0}^{\pi} \gamma^{z}(\zeta, y, u) g(\zeta, y, u, z)_{\zeta}^{\alpha-\beta-1} \sin ^{2 \beta} z d z,
\end{aligned}
$$

$1_{I_{\zeta, y}}$ being the indicator of $I_{\zeta, y}=[-|\zeta|-|y|,-|| \zeta|-| y|| U[|| \zeta|-| y||,|\zeta|+|y|]], \gamma^{z}(\zeta, y, u)=$ $1-\sigma_{\zeta, y, u}^{z}+\sigma_{u, y, \zeta}^{z}+\sigma_{u, \zeta, y}^{z}$, and

$$
\sigma_{\zeta, y, u}^{z}= \begin{cases}\frac{-\cosh u \cosh \zeta-\cosh \zeta \cosh y}{\sinh \zeta \sinh y}, & \zeta y \neq 0, \\ 0, & \zeta y=0,\end{cases}
$$

$M_{\alpha, \beta}$ being the classical function given by $[1,(1.15)]$. We denote by $D$ the set of $C^{\infty}$ functions whose supports over $\mathbb{R}$ are bounded. By $L_{\alpha, \beta}^{1}\left(\mathbb{R}, X_{\alpha, \beta}(\zeta) d \zeta\right)$, or $L_{\alpha, \beta}^{1}$, we denote the measurable space of functions over $\mathbb{R}$ that satisfies the norms [2]

$$
\|\psi\|_{\alpha, \beta}=\int_{-\infty}^{\infty}|\psi(\zeta)| X_{\alpha, \beta}(\zeta) d \zeta<\infty
$$

where

$$
X_{\alpha, \beta}(\zeta)=2^{2 \rho}(\sinh |\zeta|)^{2 \alpha+1}(\cosh \zeta)^{2 \beta+1}, \quad \rho=\alpha+\beta+1 .
$$


The convolution product of the arbitrary functions $\psi_{1}$ and $\psi_{2}$ is defined by [1, Def. (3.1)]

$$
\psi_{1} *_{\alpha, \beta} \psi_{2}(\zeta)=\int_{-\infty}^{\infty} T_{\alpha, \beta}^{\zeta}\left(\psi_{1}\right)(-y) \psi_{2}(y) X_{\alpha, \beta}(y) d y
$$

where $T_{\alpha, \beta}^{\zeta}, \zeta \in \mathbb{R}$, is the transformation operator defined by [1, Def. (2.8)]

$$
T_{\alpha, \beta}^{\zeta} \psi_{1}(y)=\mu_{\zeta(y)}^{\alpha, \beta}\left(\psi_{1}\right)=\int_{-\infty}^{\infty} \psi_{1} d \mu_{\zeta(y)}^{\alpha, \beta}
$$

However, the product $*_{\alpha, \beta}$ of the suitable functions $\psi_{1}$ and $\psi_{2}$ satisfies several results as follows [1, p. 375].

Proposition 1 Let $\psi_{1}, \psi_{2}, \psi_{3} \in L_{\alpha, \beta}^{1}\left(\mathbb{R}, X_{\alpha, \beta}(\zeta) d \zeta\right)$. Then the undermentioned relations hold true.

(i) $\psi_{1} *_{\alpha, \beta} \psi_{2}=\psi_{2} *_{\alpha, \beta} \psi_{1}$,

(ii) $\left(\psi_{1} *_{\alpha, \beta} \psi_{2}\right) *_{\alpha, \beta} \psi_{3}=\psi_{1} *_{\alpha, \beta}\left(\psi_{2} *_{\alpha, \beta} \psi_{3}\right)$.

Consequently, for $p=q=r=1$, [1, Prop. (3.2)] leads to the following fruitful result.

Proposition 2 Let $\psi_{2}, \psi_{1} \in L_{\alpha, \beta}^{1}\left(\mathbb{R}, X_{\alpha, \beta}(\zeta) d \zeta\right)$. Then the following hold:

(i) $T_{\alpha, \beta}^{x}$ is defined a.e. on $\mathbb{R}$. Moreover, it is a member of $L_{\alpha, \beta}^{1}\left(\mathbb{R}, X_{\alpha, \beta}(\zeta) d \zeta\right)$ and

$$
\left\|T_{\alpha, \beta}^{\zeta} \psi_{1}\right\|_{\alpha, \beta} \leq 4\left\|\psi_{1}\right\|_{\alpha, \beta}
$$

(ii) $\psi_{1} *_{\alpha, \beta} \psi_{2} \in L_{\alpha, \beta}^{1}\left(\mathbb{R}, X_{\alpha, \beta}(\zeta) d \zeta\right)$, and

$$
\left\|\psi_{1} *_{\alpha, \beta} \psi_{2}\right\|_{\alpha, \beta} \leq 4\left\|\psi_{1}\right\|_{\alpha, \beta}\left\|\psi_{2}\right\|_{\alpha, \beta} .
$$

The Jacobi-Dunkl operator for a suitable function $\psi_{1}$ is defined over $\mathbb{R}$ by [1, Def. 3.3]

$$
J_{\alpha, \beta}^{d}\left(\psi_{1}\right)(\lambda)=\int_{-\infty}^{\infty} \psi_{1}(\zeta) \Psi_{-\lambda}^{\alpha, \beta}(\zeta) X_{\alpha, \beta}(\zeta) d \zeta
$$

Moreover, for $\psi_{2}, \psi_{1} \in L_{\alpha, \beta}^{1}\left(\mathbb{R}, X_{\alpha, \beta}(\zeta) d \zeta\right)$ and $\lambda \in \mathbb{R}$, Prop. (3.6) of [1, p. 376] reveals

$$
J_{\alpha, \beta}^{d}\left(\psi_{1} *_{\alpha, \beta} \psi_{2}\right)(\lambda)=J_{\alpha, \beta}^{d}\left(\psi_{1}\right)(\lambda) J_{\alpha, \beta}^{d}\left(\psi_{2}\right)(\lambda)
$$

The Plancherel formula for the $J_{\alpha, \beta}^{d}$ transform is defined as

$$
\int_{-\infty}^{\infty}\left|\psi_{1}(\zeta)\right|^{2} X_{\alpha, \beta}(\zeta) d \zeta=\int_{-\infty}^{\infty}\left|J_{\alpha, \beta}^{d}\left(\psi_{1}\right)(\lambda)\right|^{2} d \Pi_{\alpha, \beta}(\lambda)
$$

where

$$
d \Pi_{\alpha, \beta}(\lambda)=\frac{|\lambda| d \lambda}{8 \pi \sqrt{\lambda^{2}-p^{2}}\left|c_{\alpha, \beta} \sqrt{\lambda^{2}-p^{2}}\right|} 1_{\mathbb{R}(-p, p)}(\lambda)
$$


is the Plancherel measure [1, p. 376]. For more illustrations about this theory, readers are referred to $[1-4,41,43]$ and [5-20] and the references cited therein. However, this research is organized in the following format. In Sect. 1, we present some definitions and results associated with the Jacobi-Dunkl function theory. In Sect. 2, we establish the generalized spaces $B\left(L_{\alpha, \beta}^{1}, D, *_{\alpha, \beta}, \Delta\right)$ and $B\left(\bar{L}_{\alpha, \beta}^{1}, \bar{D}, \times, \bar{\Delta}\right)$. In Sect. 3, we extend the JacobiDunkl function theory to the generalized spaces of generalized functions.

\section{The spaces $B\left(L_{\alpha, \beta}^{1}, D, *_{\alpha, \beta}, \Delta\right)$ and $B\left(\bar{L}_{\alpha, \beta}^{1}, \bar{D}, x, \bar{\Delta}\right)$}

The concepts of the Boehmian spaces are obtained by following an algebraic approach that hires convolutions and delta sequences, which are approximating identities. When the structure is allowed to be a function space and the space multiplication is interpreted as a convolution product, the new structure yields a space of Boehmians (see, e.g., [2, 21$35,44]$ and $[1,8,11,33,36-40,42])$. Let $\Delta$ be the set of all sequences $\left(\delta_{n}\right)$ in $D$ such that the following properties hold:

$$
\begin{aligned}
& \int_{-\infty}^{\infty} \delta_{n}(\zeta) X_{\alpha, \beta}(\zeta) d \zeta=1, \\
& \int_{-\infty}^{\infty}\left|\delta_{n}(\zeta)\right| X_{\alpha, \beta}(\zeta) d \zeta<M, \quad M \in \mathbb{R}, \\
& \operatorname{supp}\left(\delta_{n}\right) \subset\left(0, a_{n}\right), \quad a_{n} \rightarrow 0 \text { as } n \rightarrow \infty
\end{aligned}
$$

The following result shows that $\Delta$ is a set of delta sequences.

Lemma 3 Let $\left(\delta_{n}\right)$ and $\left(\theta_{n}\right)$ be in $\Delta$. Then $\left(\delta_{n} *_{\alpha, \beta} \theta_{n}\right)$ is in $\Delta$.

Proof Let $\left(\delta_{n}\right)$ and $\left(\theta_{n}\right)$ be in $\Delta$. Then, to prove this lemma, we have to prove that Eqs. (10)(12) hold for $\left(\delta_{n} *_{\alpha, \beta} \theta_{n}\right)$. By [1, Eq. (2.2)] we infer that $\psi_{\lambda}^{\alpha, \beta}(\zeta)=1$ for $\lambda=0$. Therefore, we obtain

$$
J_{\alpha, \beta}^{d}\left(\psi_{1}\right)(0)=\int_{-\infty}^{\infty} \psi_{1}(\zeta) \Psi_{0}^{\alpha, \beta}(\zeta) X_{\alpha, \beta}(\zeta) d \zeta=\int_{-\infty}^{\infty} \psi_{1}(\zeta) X_{\alpha, \beta}(\zeta) d \zeta
$$

Hence, by the convolution theorem, Eq. (9) and Eq. (10) give

$$
\begin{aligned}
J_{\alpha, \beta}^{d}\left(\delta_{n} *_{\alpha, \beta} \theta_{n}\right)(0) & =J_{\alpha, \beta}^{d}\left(\delta_{n}\right)(0) J_{\alpha, \beta}^{d}\left(\theta_{n}\right)(0) \\
& =\int_{-\infty}^{\infty} \delta_{n}(\zeta) X_{\alpha, \beta}(\zeta) d \zeta \int_{-\infty}^{\infty} \theta_{n}(y) X_{\alpha, \beta}(y) d y \\
& =1 .
\end{aligned}
$$

This proves that Eq. (10) holds for $\left(\delta_{n} *_{\alpha, \beta} \theta_{n}\right)$. To show that Eq. (11) holds for $\left(\delta_{n} *_{\alpha, \beta} \theta_{n}\right)$, we use Proposition 2 to obtain

$$
\left\|\delta_{n} *_{\alpha, \beta} \theta_{n}\right\|_{\alpha, \beta} \leq 4\left\|\delta_{n}\right\|_{\alpha, \beta}\left\|\theta_{n}\right\|_{\alpha, \beta} \leq 4 M^{2}
$$

Finally, the proof of the fact that $\left(\delta_{n} *_{\alpha, \beta} \theta_{n}\right)$ satisfies Eq. (12) is straightforward. The proof is therefore completed.

Hence, the necessary axioms for establishing the Boehmians space $B\left(L_{\alpha, \beta}^{1}, D, *_{\alpha, \beta}, \Delta\right)$ : 
(i) $\psi_{1} *_{\alpha, \beta} \psi_{2}=\psi_{2} *_{\alpha, \beta} \psi_{1}, \psi_{1} \in L_{\alpha, \beta}^{1}$ and $\psi_{2} \in D$;

(ii) $\psi_{1} *_{\alpha, \beta}\left(\psi_{2} *_{\alpha, \beta} \psi_{3}\right)=\left(\psi_{1} *_{\alpha, \beta} \psi_{2}\right) *_{\alpha, \beta} \psi_{3}, \psi_{1} \in L_{\alpha, \beta}^{1}$ and $\psi_{2}, \psi_{3} \in D$;

(iii) $\psi_{1} *_{\alpha, \beta} \psi_{2} \in L_{\alpha, \beta}^{1}, \psi_{1} \in L_{\alpha, \beta}^{1}, \psi_{2} \in D$,

are justified by Propositions 1 and 2 . Hence, we omit the details.

Theorem 4 Let $\psi_{3}, \psi_{1}, \psi_{n} \in L_{\alpha, \beta}^{1}$ and $\psi_{2} \in D$. Then the undermentioned relations hold.

(i) $\psi_{n} *_{\alpha, \beta} \psi_{2} \rightarrow \psi_{1} *_{\alpha, \beta} \psi_{2}$ as $n \rightarrow \infty$ as $\psi_{n} \rightarrow \psi_{1}$.

(ii) $\left(\psi_{1}+\psi_{3}\right) *_{\alpha, \beta} \psi_{2}=\psi_{1} *_{\alpha, \beta} \psi_{2}+\psi_{3} *_{\alpha, \beta} \psi_{2}$.

Proof of this lemma can be easily obtained from using Eq. (6). Hence, the details are deleted.

To complete the process of establishing the space $B\left(L_{\alpha, \beta}^{1}, D, *_{\alpha, \beta}, \Delta\right)$, we derive the following relation.

Lemma 5 Let $\left(\delta_{n}\right) \in \Delta$ and $\psi \in L_{\alpha, \beta}^{1}$. Then we have $\psi *_{\alpha, \beta} \delta_{n} \rightarrow \psi$ as $n \rightarrow \infty$.

Proof It has already been verified that $\psi_{1} *_{\alpha, \beta} \delta_{n} \in L_{\alpha, \beta}^{1}$. Therefore, from definitions we get

$$
\left\|\psi *_{\alpha, \beta} \delta_{n}-\psi\right\|_{\alpha, \beta}=\int_{-\infty}^{\infty}\left|\left(\psi *_{\alpha, \beta} \delta_{n}-\psi\right)(\zeta)\right| X_{\alpha, \beta}(\zeta) d \zeta
$$

Thus, by employing Eq. (10), we obtain

$$
\left\|\psi *_{\alpha, \beta} \delta_{n}-\psi\right\|_{\alpha, \beta} \leq \int_{-\infty}^{\infty}\left|\delta_{n}(y)\right|\left|T_{\alpha, \beta}^{\zeta} \psi(-y)-\psi(\zeta)\right| X_{\alpha, \beta}(\zeta) d \zeta X_{\alpha, \beta}(y) d y .
$$

Again by Part (i) of Proposition 2, Eq. (13) gives

$$
\left\|\psi *_{\alpha, \beta} \delta_{n}-\psi\right\|_{\alpha, \beta} \leq\left(4\|\psi\|_{\alpha, \beta}-\|\psi\|_{\alpha, \beta}\right) \int_{-\infty}^{\infty}\left|\delta_{n}(y) X_{\alpha, \beta}(y) d y\right| .
$$

Since $\left(\delta_{n}\right)$ is of compact support, we by Eq. (12) get

$$
\left\|\psi *_{\alpha, \beta} \delta_{n}-\psi\right\|_{\alpha, \beta} \leq 3\|\psi\|_{\alpha, \beta} A a_{n} \rightarrow 0
$$

as $n \rightarrow \infty$.

This ends the proof of the lemma.

Therefore the Boehmian space $B\left(L_{\alpha, \beta}^{1}, D, *_{\alpha, \beta}, \Delta\right)$ is obtained. The sum of the Boehmians $\left[\frac{\varphi_{n}}{\delta_{n}}\right]$ and $\left[\frac{\psi_{n}}{\varepsilon_{n}}\right]$ is given in $B\left(L_{\alpha, \beta}^{1}, D, *_{\alpha, \beta}, \Delta\right)$ as

$$
\left[\frac{\varphi_{n}}{\delta_{n}}\right]+\left[\frac{\psi_{n}}{\varepsilon_{n}}\right]=\left[\frac{\varphi_{n} *_{\alpha, \beta} \delta_{n}+\psi_{n} *_{\alpha, \beta} \delta_{n}}{\delta_{n} *_{\alpha, \beta} \varepsilon_{n}}\right]
$$

whereas the multiplication of a Boehmian $\frac{\varphi_{n}}{\delta_{n}}$ or sometimes $\left[\frac{\varphi_{n}}{\delta_{n}}\right]$ in $B\left(L_{\alpha, \beta}^{1}, D, *_{\alpha, \beta}, \Delta\right)$ by a complex number $\gamma$ is defined as $\gamma\left[\frac{\varphi_{n}}{\delta_{n}}\right]=\left[\frac{\gamma \varphi_{n}}{\delta_{n}}\right]$. On the other hand, the extension of $*_{\alpha, \beta}$ and $D^{\alpha}$ to $B\left(L_{\alpha, \beta}^{1}, D, *_{\alpha, \beta}, \Delta\right)$ is introduced as

$$
\left[\frac{\varphi_{n}}{\delta_{n}} *_{\alpha, \beta} \frac{\psi_{n}}{\varepsilon_{n}}\right]=\left[\frac{\varphi_{n} *_{\alpha, \beta} \psi_{n}}{\delta_{n} *_{\alpha, \beta} \varepsilon_{n}}\right] \quad \text { and } \quad D^{\alpha}\left[\frac{\varphi_{n}}{\delta_{n}}\right]=\left[\frac{D^{\alpha} \varphi_{n}}{\delta_{n}}\right], \quad \alpha \in \mathbb{R}
$$


Moreover, an extension of $*_{\alpha, \beta}$ to $B\left(L_{\alpha, \beta}^{1}, D, *_{\alpha, \beta}, \Delta\right) *_{\alpha, \beta} L_{\alpha, \beta}^{1}\left(\mathbb{R}^{2}\right)$, where $\left(\varphi_{n} / \delta_{n}\right)$ is in $B\left(L_{\alpha, \beta}^{1}, D, *_{\alpha, \beta}, \Delta\right)$ and $\omega$ in $L_{\alpha, \beta}^{1}$, is given as

$$
\left[\frac{\varphi_{n}}{\delta_{n}} *_{\alpha, \beta} \omega\right]=\left[\frac{\varphi_{n} *_{\alpha, \beta} \omega}{\delta_{n}}\right] .
$$

If $\beta_{n}, \beta \in B\left(L_{\alpha, \beta}^{1}, D, *_{\alpha, \beta}, \Delta\right), n=1,2,3, \ldots$, then $\left\{\beta_{n}\right\}$ converges in $\delta$ type to $\beta$, namely $\delta-$ $\lim _{n \rightarrow \infty} \beta_{n}=\beta\left(\beta_{n} \stackrel{\delta}{\rightarrow} \beta\right)$, provided there can be found a delta sequence $\left\{\delta_{n}\right\}$ such that

(a) $\left(\beta_{n} *_{\alpha, \beta} \delta_{k}\right)$ and $\left(\beta *_{\alpha, \beta} \delta_{k}\right) \in L_{\alpha, \beta}^{1}$ for all $n, k \in \mathbb{N}$,

(b) $\lim _{n \rightarrow \infty} \beta_{n} *_{\alpha, \beta} \delta_{k}=\beta *_{\alpha, \beta} \delta_{k}$ in $L_{\alpha, \beta}^{1}$ for every $k \in \mathbb{N}$.

Or, equivalently, $\delta-\lim _{n \rightarrow \infty} \beta_{n}=\beta$ if and only if there are $\varphi_{n, k}, \varphi_{k} \in L_{\alpha, \beta}^{1}$ and $\left\{\delta_{k}\right\} \in \Delta$ such that

(i) $\beta_{n}=\varphi_{n, k} / \delta_{k}, \beta=\varphi_{k} / \delta_{k}$

(ii) $\lim _{n \rightarrow \infty} \varphi_{n, k}=\varphi_{k} \in L_{\alpha, \beta}^{1}$ to every $k \in \mathbb{N}$.

If $\beta_{n}, \beta \in B\left(L_{\alpha, \beta}^{1}, D, *_{\alpha, \beta}, \Delta\right)$ for $n=1,2,3, \ldots$, then the sequence $\left\{\beta_{n}\right\}$ converges in $\Delta$ type to $\beta$, namely $\Delta$ - $\lim _{n \rightarrow \infty} \beta_{n}=\beta\left(\beta_{n} \stackrel{\Delta}{\rightarrow} \beta\right)$, provided there can be found a delta sequence $\left\{\delta_{n}\right\}$ such that

(i) $\left(\beta_{n}-\beta\right) *_{\alpha, \beta} \delta_{n} \in L_{\alpha, \beta}^{1}(\forall n \in \mathbb{N})$

(ii) $\lim _{n \rightarrow \infty}\left(\beta_{n}-\beta\right) *_{\alpha, \beta} \delta_{n}=0$ in $L_{\alpha, \beta}^{1}$.

We turn to the construction of the ultraspace $B\left(\bar{L}_{\alpha, \beta}^{1}, \bar{D}, \times, \bar{\Delta}\right)$ of Boehmians. Let $\bar{D}, \bar{L}_{\alpha, \beta}^{1}$ be the spaces of all Jacobi-Dunkl transforms of the spaces $D$ and $L_{\alpha, \beta}^{1}$, respectively, and $\bar{\Delta}$ be the set of the Jacobi-Dunkl transforms of the set $\Delta$. Define a product formula $\times_{\alpha, \beta}$ between $\bar{D}$ and $\bar{L}_{\alpha, \beta}^{1}$ by

$$
F \times_{\alpha, \beta} G=\left(J_{\alpha, \beta}^{d} \psi_{1}\right)\left(J_{\alpha, \beta}^{d} \psi_{2}\right), \quad \text { where } \psi_{1} \in D \text { and } \psi_{2} \in L_{\alpha, \beta}^{1} .
$$

With the product $\times_{\alpha, \beta}$, the space $B\left(\bar{L}_{\alpha, \beta}^{1}, \bar{D}, \times_{\alpha, \beta}, \bar{\Delta}\right)$ can be easily verified as a Boehmian space by virtue of the following result.

Theorem 6 Let $F_{1}, F_{2} \in \bar{L}_{\alpha, \beta}^{1}, G_{1}, G \in \bar{D}$. Then the undermentioned relations hold.

(i) $\left(F_{1}+F_{2}\right) \times_{\alpha, \beta} G=F_{1} \times_{\alpha, \beta} G+F_{2} \times_{\alpha, \beta} G$.

(ii) $F_{1} \times_{\alpha, \beta} G=G \times_{\alpha, \beta} F_{1}$.

(iii) $\lambda\left(F_{1} \times_{\alpha, \beta} G\right)=\left(\lambda F_{1}\right) \times_{\alpha, \beta} G, \lambda \in \mathbb{C}$.

(iv) $F_{n} \times_{\alpha, \beta} G \rightarrow F_{1} \times_{\alpha, \beta} G$ for every $F_{n} \in \bar{L}_{\alpha, \beta}^{1}$.

(v) $F_{n} \rightarrow F_{1}$ as $n \rightarrow \infty$ in $\bar{L}_{\alpha, \beta}^{1}$.

(vi) $F_{1} \times_{\alpha, \beta}\left(G \times_{\alpha, \beta} G_{1}\right)=\left(F_{1} \times_{\alpha, \beta} G\right) \times_{\alpha, \beta} G_{1}$.

Proof We prove (ii) as the proofs of (i), (iii), (iv), and (v) are similar to those given in literature or are straightforward results from simple integration. Let $\psi_{1} \in L_{\alpha, \beta}^{1}$ and $\psi_{2} \in D$ be such that $J_{\alpha, \beta}^{d} \psi_{1}=F_{1}$ and $J_{\alpha, \beta}^{d} \psi_{2}=G$. Then, by Eq. (9), we write

$$
F_{1} \times_{\alpha, \beta} G=J_{\alpha, \beta}^{d} \psi_{1} \times_{\alpha, \beta} J_{\alpha, \beta}^{d} \psi_{2}=J_{\alpha, \beta}^{d}\left(\psi_{1} *_{\alpha, \beta} \psi_{2}\right) .
$$

Hence, since $\psi_{1} *_{\alpha, \beta} \psi_{2}=\psi_{2} *_{\alpha, \beta} \psi_{1}$, we have

$$
F_{1} \times G=J_{\alpha, \beta}^{d}\left(\psi_{2} *_{\alpha, \beta} \psi_{1}\right)=G \times_{\alpha, \beta} F_{1}
$$

This ends the proof of the theorem. 
Theorem 7 Let $\left(\bar{\delta}_{n}\right),\left(\bar{\theta}_{n}\right) \in \bar{\Delta}$. Then $\bar{\delta}_{n} \times_{\alpha, \beta} \bar{\theta}_{n} \in \bar{\Delta}$ for all $n \in \mathbb{N}$.

Proof Let $\left(\delta_{n}\right),\left(\theta_{n}\right)$ be in $\Delta$ such that $\bar{\delta}_{n}=J_{\alpha, \beta}^{d} \delta_{n}$ and $\bar{\theta}_{n}=J_{\alpha, \beta}^{d} \theta_{n}$. Then, by Eq. (9), we have

$$
\bar{\delta}_{n} \times_{\alpha, \beta} \bar{\theta}_{n}=J_{\alpha, \beta}^{d}\left(\delta_{n} *_{\alpha, \beta}, \theta_{n}\right)
$$

It is perspicuous that $\bar{\delta}_{n} \times_{\alpha, \beta} \bar{\theta}_{n} \in \bar{\Delta}$ as $\left(\delta_{n} *_{\alpha, \beta}, \theta_{n}\right) \in \Delta$ by Lemma 3 . This completes the proof of the theorem.

Similarly, we proceed to establishing the following theorem.

Theorem 8 Let $\left(\bar{\delta}_{n}\right) \in \bar{\Delta}$ and $F \in \bar{L}_{\alpha, \beta}^{1}$. Then we have

$$
F \times_{\alpha, \beta} \bar{\delta}_{n} \rightarrow F \quad \text { as } n \rightarrow \infty
$$

The space $B\left(\bar{L}_{\alpha, \beta}^{1}, \bar{D}, \times_{\alpha, \beta}, \bar{\Delta}\right)$ is an ultra-Boehmian space. For addition, multiplication by a scalar, $\delta$-convergence, and $\Delta$-convergence in the space $B\left(\bar{L}_{\alpha, \beta}^{1}, \bar{D}, \times_{\alpha, \beta}, \bar{\Delta}\right)$, see $[2,21-32]$ and $B\left(L_{\alpha, \beta}^{1}, D, *_{\alpha, \beta}, \Delta\right)$ for similar definitions, replacing $*_{\alpha, \beta}$ with $\times_{\alpha, \beta}$.

\section{The generalized Jacobi-Dunkl transform}

In this section, we aim to introduce the generalized definition of the Jacobi-Dunkl integral operator. Let $\left[\frac{\psi_{n}}{\delta_{n}}\right] \in B\left(L_{\alpha, \beta}^{1}, D, *_{\alpha, \beta}, \Delta\right)$, then the generalized Jacobi-Dunkl transform of $\left[\frac{\psi_{n}}{\delta_{n}}\right]$ is a Boehmian in $B\left(\bar{L}_{\alpha, \beta}^{1}, \bar{D}, \times_{\alpha, \beta}, \bar{\Delta}\right)$ defined by

$$
\bar{F}_{\alpha, \beta}\left[\frac{\psi_{n}}{\delta_{n}}\right]=\left[\frac{J_{\alpha, \beta}^{d} \psi_{n}}{J_{\alpha, \beta}^{d} \bar{\delta}_{n}}\right]
$$

Theorem 9 The estimated generalized Jacobi-Dunkl operator $\bar{F}_{\alpha, \beta}$ is well defined and linear from the space $B\left(L_{\alpha, \beta}^{1}, D, *_{\alpha, \beta}, \Delta\right)$ into the space $B\left(\bar{L}_{\alpha, \beta}^{1}, \bar{D}, \times_{\alpha, \beta}, \bar{\Delta}\right)$.

Proof Let $\left[\frac{\varphi_{n}}{\delta_{n}}\right]=\left[\frac{\psi_{n}}{\varepsilon_{n}}\right] \in B\left(L_{\alpha, \beta}^{1}, D, *_{\alpha, \beta}, \Delta\right)$. Then we have

$$
\varphi_{n} *_{\alpha, \beta} \varepsilon_{m}=\psi_{m} *_{\alpha, \beta} \delta_{n}=\psi_{n} *_{\alpha, \beta} \delta_{m} \quad \text { for all } m, n \in \mathbb{N}
$$

Applying $\bar{F}_{\alpha, \beta}$ to both sides in the preceding equation and making use of Eq. (9) reveal that

$$
J_{\alpha, \beta}^{d} \varphi_{n} \times_{\alpha, \beta} J_{\alpha, \beta}^{d} \varepsilon_{m}=J_{\alpha, \beta}^{d} \psi_{n} \times_{\alpha, \beta} J_{\alpha, \beta}^{d} \delta_{m} \quad \text { for all } m, n \in \mathbb{N} .
$$

In view of the concept of quotients of the sequences of $B\left(\bar{L}_{\alpha, \beta}^{1}, \bar{D}, \times_{\alpha, \beta}, \bar{\Delta}\right)$, Eq. (16) gives

$$
\left[\frac{J_{\alpha, \beta}^{d} \varphi_{n}}{J_{\alpha, \beta}^{d} \delta_{n}}\right]=\left[\frac{J_{\alpha, \beta}^{d} \psi_{n}}{J_{\alpha, \beta}^{d} \varepsilon_{n}}\right] .
$$

To show that the transform $\bar{F}_{\alpha, \beta}: B\left(L_{\alpha, \beta}^{1}, D, *_{\alpha, \beta}, \Delta\right) \rightarrow B\left(\bar{L}_{\alpha, \beta}^{1}, \bar{D}, \times_{\alpha, \beta}, \bar{\Delta}\right)$ is linear, let $\left[\frac{\varphi_{n}}{\delta_{n}}\right],\left[\frac{\psi_{n}}{\varepsilon_{n}}\right] \in B\left(L_{\alpha, \beta}^{1}, D, *_{\alpha, \beta}, \Delta\right)$. Then, by the idea of the addition of $B\left(L_{\alpha, \beta}^{1}, D, *_{\alpha, \beta}, \Delta\right)$, Eq. (15), Eq. (9), and the idea of the addition of $B\left(\bar{L}_{\alpha, \beta}^{1}, \bar{D}, \times_{\alpha, \beta}, \bar{\Delta}\right)$, we can announce that

$$
\bar{F}_{\alpha, \beta}\left(\left[\frac{\varphi_{n}}{\delta_{n}}\right]+\left[\frac{\psi_{n}}{\varepsilon_{n}}\right]\right)=\bar{F}_{\alpha, \beta}\left(\left[\frac{\varphi_{n} *_{\alpha, \beta} \varepsilon_{n}+\psi_{n} *_{\alpha, \beta} \delta_{n}}{\delta_{n} *_{\alpha, \beta} \varepsilon_{n}}\right]\right)
$$




$$
\begin{aligned}
& =\left[\frac{J_{\alpha, \beta}^{d}\left(\varphi_{n} *_{\alpha, \beta} \varepsilon_{n}\right)+J_{\alpha, \beta}^{d}\left(\psi_{n} *_{\alpha, \beta} \delta_{n}\right)}{J_{\alpha, \beta}^{d} \delta_{n} \times_{\alpha, \beta} J_{\alpha, \beta}^{d} \varepsilon_{n}}\right] \\
& =\left[\frac{J_{\alpha, \beta}^{d} \varphi_{n} \times_{\alpha, \beta} J_{\alpha, \beta}^{d} \varepsilon_{n}+J_{\alpha, \beta}^{d} \psi_{n} \times_{\alpha, \beta} J_{\alpha, \beta}^{d} \delta_{n}}{J_{\alpha, \beta}^{d} r_{n} \times_{\alpha, \beta} J_{\alpha, \beta}^{d} \varepsilon_{n}}\right] \\
& =\left[\frac{J_{\alpha, \beta}^{d} \varphi_{n}}{J_{\alpha, \beta}^{d} \delta_{n}}\right]+\left[\frac{J_{\alpha, \beta}^{d} \psi_{n}}{J_{\alpha, \beta}^{d} \varepsilon_{n}}\right] .
\end{aligned}
$$

Hence, Eq. (15) leads to

$$
\bar{F}_{\alpha, \beta}\left(\left[\frac{\varphi_{n}}{\delta_{n}}\right]+\left[\frac{\psi_{n}}{\varepsilon_{n}}\right]\right)=\bar{F}_{\alpha, \beta}\left[\frac{\varphi_{n}}{\delta_{n}}\right]+\bar{F}_{\alpha, \beta}\left[\frac{\psi_{n}}{\varepsilon_{n}}\right] .
$$

Also, we have

$$
\alpha \bar{F}_{\alpha, \beta}\left[\frac{\varphi_{n}}{\delta_{n}}\right]=\alpha\left[\frac{J_{\alpha, \beta}^{d} \varphi_{n}}{J_{\alpha, \beta}^{d} \delta_{n}}\right]=\left[\frac{J_{\alpha, \beta}^{d}\left(\alpha \varphi_{n}\right)}{J_{\alpha, \beta}^{d} \delta_{n}}\right] \quad \text { for } \alpha \in \mathbb{C} .
$$

Hence,

$$
\alpha \bar{F}_{\alpha, \beta}\left[\frac{\varphi_{n}}{\delta_{n}}\right]=\bar{F}_{\alpha, \beta}\left(\alpha\left[\frac{\varphi_{n}}{\delta_{n}}\right]\right) .
$$

Equations (17) and (18) end the proof of this theorem.

Theorem 10 The mapping $\bar{F}_{\alpha, \beta}: B\left(L_{\alpha, \beta}^{1}, D, *_{\alpha, \beta}, \Delta\right) \rightarrow B\left(\bar{L}_{\alpha, \beta}^{1}, \bar{D}, \times_{\alpha, \beta}, \bar{\Delta}\right)$ is an isomorphism.

Proof Let $\left[\frac{J_{\alpha, \beta}^{d} \varphi_{n}}{\delta_{n}}\right]=\left[\frac{J_{\alpha, \beta}^{d} \psi_{n}}{\varepsilon_{n}}\right] \in B\left(\bar{L}_{\alpha, \beta}^{1}, \bar{D}, \times_{\alpha, \beta}, \bar{\Delta}\right)$. Then, by using Eq. (9), we get

$$
J_{\alpha, \beta}^{d} \varphi_{n} \times_{\alpha, \beta} J_{\alpha, \beta}^{d} \varepsilon_{m}=J_{\alpha, \beta}^{d} \psi_{m} \times_{\alpha, \beta} J_{\alpha, \beta}^{d} \delta_{n} \quad \text { for all } m, n \in \mathbb{N} \text {. }
$$

Once again, (14) reveals to have

$$
J_{\alpha, \beta}^{d}\left(\varphi_{n} *_{\alpha, \beta} \varepsilon_{m}\right)=J_{\alpha, \beta}^{d}\left(\psi_{m} *_{\alpha, \beta} \delta_{n}\right) \quad \text { for all } m, n \in \mathbb{N} .
$$

We, thus, obtain $\varphi_{n} *_{\alpha, \beta} \varepsilon_{m}=\psi_{m} *_{\alpha, \beta} \delta_{n}$ for all $m, n \in \mathbb{N}$. Hence, by the concept of quotients of $B\left(L_{\alpha, \beta}^{1}, D, *_{\alpha, \beta}, \Delta\right)$, we have

$$
\left[\frac{\varphi_{n}}{\delta_{n}}\right]=\left[\frac{\psi_{n}}{\varepsilon_{n}}\right] \in B\left(L_{\alpha, \beta}^{1}, D, *_{\alpha, \beta}, \Delta\right) .
$$

This confirms the injectivity of the mapping. The surjectivity part of $\bar{F}_{\alpha, \beta}$ is very clear as, for every $\left[\frac{J_{\alpha, \beta}^{d} \varphi_{n}}{J_{\alpha, \beta}^{d} \delta_{n}}\right] \in B\left(\bar{L}_{\alpha, \beta}^{1}, \bar{D}, \times_{\alpha, \beta}, \bar{\Delta}\right)$, there can be found $\left[\frac{\varphi_{n}}{\delta_{n}}\right] \in B\left(\bar{L}_{\alpha, \beta}^{1}, \bar{D}, \times_{\alpha, \beta}, \bar{\Delta}\right)$ such that

$$
\bar{F}_{\alpha, \beta}\left[\frac{\varphi_{n}}{\delta_{n}}\right]=\left[\frac{J_{\alpha, \beta}^{d} \varphi_{n}}{J_{\alpha, \beta}^{d} \delta_{n}}\right]
$$

The proof of the theorem is ended. 
Definition 11 Let $\left[\frac{J_{\alpha, \beta}^{d} \varphi_{n}}{J_{\alpha, \beta}^{d} \delta_{n}}\right] \in B\left(\bar{L}_{\alpha, \beta}^{1}, \bar{D}, \times_{\alpha, \beta}, \bar{\Delta}\right)$. Then we define the transform inversion formula of $\bar{F}_{\alpha, \beta}$ as

$$
\left(\bar{F}_{\alpha, \beta}\right)^{-1}\left[\frac{J_{\alpha, \beta}^{d} \varphi_{n}}{J_{\alpha, \beta}^{d} \delta_{n}}\right]=\left[\frac{\varphi_{n}}{\delta_{n}}\right] \text { for each }\left\{\delta_{n}\right\} \in \Delta
$$

Theorem 12 Let $\left[\frac{\alpha_{\alpha, \beta}^{d} \varphi_{n}}{J_{\alpha, \beta}^{d} \delta_{n}}\right]$ be in $B\left(\bar{L}_{\alpha, \beta}^{1}, \bar{D}, \times_{\alpha, \beta}, \bar{\Delta}\right)$ for some $\left[\frac{\varphi_{n}}{\delta_{n}}\right]$ in $B\left(L_{\alpha, \beta}^{1}, D, *_{\alpha, \beta}, \Delta\right)$. Then, for $\phi \in \bar{D}(\mathbb{R})$ and $\psi \in D$, we have

$$
\begin{aligned}
& \left(\bar{F}_{\alpha, \beta}\right)^{-1}\left(\left[\frac{J_{\alpha, \beta}^{d} \varphi_{n}}{J_{\alpha, \beta}^{d} \delta_{n}}\right] \times_{\alpha, \beta} \phi\right)=\left[\frac{\varphi_{n}}{\delta_{n}}\right] *_{\alpha, \beta} \theta \text { and } \\
& \bar{F}_{\alpha, \beta}\left(\left[\frac{\varphi_{n}}{\delta_{n}}\right] *_{\alpha, \beta} \psi\right)=\left[\frac{J_{\alpha, \beta}^{d} \varphi_{n}}{J_{\alpha, \beta}^{d} \delta_{n}}\right] \times_{\alpha, \beta} \psi
\end{aligned}
$$

for some $\theta \in D$.

Proof Let $\left[\frac{J_{\alpha, \beta}^{d} \varphi_{n}}{J_{\alpha, \beta}^{d} \delta_{n}}\right] \in B\left(\bar{L}_{\alpha, \beta}^{1}, \bar{D}, \times_{\alpha, \beta}, \bar{\Delta}\right)$ and $\phi \in \bar{D}(\mathbb{R})$ be such that $\phi=J_{\alpha, \beta}^{d} \theta$ for some $\theta \in D$. Then, by Eq. (9), we write

$$
\begin{aligned}
\left(\bar{F}_{\alpha, \beta}\right)^{-1}\left(\left[\frac{J_{\alpha, \beta}^{d} \varphi_{n}}{J_{\alpha, \beta}^{d} \delta_{n}}\right] \times_{\alpha, \beta} \phi\right) & =\left(\bar{F}_{\alpha, \beta}\right)^{-1}\left(\left[\frac{J_{\alpha, \beta}^{d} \varphi_{n} \times_{\alpha, \beta} \phi}{J_{\alpha, \beta}^{d} \delta_{n}}\right]\right) \\
& =\left[\frac{\left(J_{\alpha, \beta}^{d}\right)^{-1}\left(J_{\alpha, \beta}^{d} \varphi_{n} \times_{\alpha, \beta} J_{\alpha, \beta}^{d} \theta\right)}{\left(J_{\alpha, \beta}^{d}\right)^{-1}\left(J_{\alpha, \beta}^{d} \delta_{n}\right)}\right] \\
& =\left(J_{\alpha, \beta}^{d}\right)^{-1}\left[\frac{J_{\alpha, \beta}^{d}\left(\varphi_{n} *_{\alpha, \beta} \theta\right)}{J_{\alpha, \beta}^{d} \delta_{n}}\right] \\
& =\left[\frac{\varphi_{n}}{\delta_{n}}\right] *_{\alpha, \beta} \theta .
\end{aligned}
$$

To prove the second identity of this theorem, we make use of Eq. (9) to obtain

$$
\bar{F}_{\alpha, \beta}\left(\left[\frac{\varphi_{n}}{\delta_{n}}\right] *_{\alpha, \beta} \psi\right)=\bar{F}_{\alpha, \beta}\left(\left[\frac{\varphi_{n} *_{\alpha, \beta} \psi}{\delta_{n}}\right]\right)=\left[\frac{J_{\alpha, \beta}^{d} \varphi_{n}}{J_{\alpha, \beta}^{d} \delta_{n}}\right] \times_{\alpha, \beta} \psi
$$

This ends the proof of the theorem.

Theorem 13 The mappings $\bar{F}_{\alpha, \beta}: B\left(L_{\alpha, \beta}^{1}, D, *_{\alpha, \beta}, \Delta\right) \rightarrow B\left(\bar{L}_{\alpha, \beta}^{1}, \bar{D}, \times_{\alpha, \beta}, \bar{\Delta}\right)$ and $\left(\bar{F}_{\alpha, \beta}\right)^{-1}$ : $B\left(\bar{L}_{\alpha, \beta}^{1}, \bar{D}, \times_{\alpha, \beta}, \bar{\Delta}\right) \rightarrow B\left(L_{\alpha, \beta}^{1}, D, *_{\alpha, \beta}, \Delta\right)$ are continuous with respect to $\delta$ and $\Delta$ convergence.

Proof We show that $\bar{F}_{\alpha, \beta}$ and $\left(\bar{F}_{\alpha, \beta}\right)^{-1}$ are continuous with respect to the convergence of $\delta$ type. For this aim, we assume $\beta_{n} \stackrel{\delta}{\rightarrow} \beta$ in $B\left(L_{\alpha, \beta}^{1}, D, *_{\alpha, \beta}, \Delta\right)$ as $n \rightarrow \infty$ and verify that $\bar{F}_{\alpha, \beta} \beta_{n} \rightarrow \bar{F}_{\alpha, \beta} \beta$ as $n \rightarrow \infty$. Let $\psi_{n, k}$ and $\psi_{k}$ be in $L_{\alpha, \beta}^{1}$ such that

$$
\beta_{n}=\left[\frac{\psi_{n, k}}{\phi_{k}}\right] \text { and } \beta=\left[\frac{\psi_{k}}{\phi_{k}}\right]
$$


and $\psi_{n, k} \rightarrow \psi_{k}$ as $n \rightarrow \infty$ for all $k \in \mathbb{N}$. Then $J_{\alpha, \beta}^{d} \psi_{n, k} \rightarrow J_{\alpha, \beta}^{d} \psi_{k}$ as $n \rightarrow \infty$ in the space $\bar{L}_{\alpha, \beta}^{1}$. Therefore,

$$
\left[\frac{J_{\alpha, \beta}^{d} \psi_{n, k}}{J_{\alpha, \beta}^{d} \phi_{k}}\right] \rightarrow\left[\frac{J_{\alpha, \beta}^{d} \psi_{k}}{J_{\alpha, \beta}^{d} \phi_{k}}\right]
$$

as $n \rightarrow \infty$ in $B\left(\bar{L}_{\alpha, \beta}^{1}, \bar{D}, \times_{\alpha, \beta}, \bar{\Delta}\right)$.

To prove the second part, let $g_{n} \stackrel{\delta}{\rightarrow} g$ in $B\left(\bar{L}_{\alpha, \beta}^{1}, \bar{D}, \times_{\alpha, \beta}, \bar{\Delta}\right)$ as $n \rightarrow \infty$. Then, let $g_{n}=$ $\left[\frac{\int_{\alpha, \beta}^{d} \psi_{n, k}}{J_{\alpha, \beta}^{d} \phi_{k}}\right]$ and $g=\left[\frac{\int_{\alpha, \beta}^{d} \psi_{k}}{\int_{\alpha, \beta}^{d} \phi_{k}}\right]$ and $J_{\alpha, \beta}^{d} \psi_{n, k} \rightarrow J_{\alpha, \beta}^{d} \psi_{k}$ as $n \rightarrow \infty$. Therefore, $\psi_{n, k} \rightarrow \psi_{k}$ in $B\left(L_{\alpha, \beta}^{1}, D, *_{\alpha, \beta}, \Delta\right)$ as $n \rightarrow \infty$. Hence, $\left[\frac{\psi_{n, k}}{\phi_{k}}\right] \rightarrow\left[\frac{\psi_{k}}{\phi_{k}}\right]$ as $n \rightarrow \infty$. Using Eq. (15) reveals

$$
\left(\bar{F}_{\alpha, \beta}\right)^{-1}\left[\frac{J_{\alpha, \beta}^{d} \psi_{n, k}}{J_{\alpha, \beta}^{d} \phi_{k}}\right] \rightarrow\left(\bar{F}_{\alpha, \beta}\right)^{-1}\left[\frac{J_{\alpha, \beta}^{d} \psi_{k}}{J_{\alpha, \beta}^{d} \phi_{k}}\right] \text { as } n \rightarrow \infty .
$$

To establish continuity with respect to the convergence of $\Delta$ type, we assume $\beta_{n} \stackrel{\Delta}{\rightarrow} \beta$ in $B\left(L_{\alpha, \beta}^{1}, D, *_{\alpha, \beta}, \Delta\right)$ as $n \rightarrow \infty$. Then there exist $\psi_{n} \in L_{\alpha, \beta}^{1}$ and $\left(\phi_{n}\right) \in \Delta$ such that $\left(\beta_{n}-\beta\right) *_{\alpha, \beta}$ $\phi_{n}=\left[\frac{\psi_{n} *_{\alpha}, \beta \phi_{k}}{\phi_{k}}\right]$ and $\psi_{n} \rightarrow 0$ as $n \rightarrow \infty$. Employing (15) gives

$$
\bar{F}_{\alpha, \beta}\left(\left(\beta_{n}-\beta\right) *_{\alpha, \beta} \phi_{n}\right)=\left[\frac{J_{\alpha, \beta}^{d}\left(\psi_{n} *_{\alpha, \beta} \phi_{k}\right)}{J_{\alpha, \beta}^{d} \phi_{k}}\right] .
$$

Hence, we derive

$$
\bar{F}_{\alpha, \beta}\left(\left(\beta_{n}-\beta\right) *_{\alpha, \beta} \phi_{n}\right)=\left[\frac{J_{\alpha, \beta}^{d} \psi_{n} \times_{\alpha, \beta} J_{\alpha, \beta}^{d} \phi_{k}}{J_{\alpha, \beta}^{d} \phi_{k}}\right] \simeq J_{\alpha, \beta}^{d} f_{n} \rightarrow 0
$$

as $n \rightarrow \infty$ in $\bar{L}_{\alpha, \beta}^{1}$. Therefore, from Eq. (22) we get

$$
\bar{F}_{\alpha, \beta}\left(\left(\beta_{n}-\beta\right) *_{\alpha, \beta} \phi_{n}\right)=\left(\bar{F}_{\alpha, \beta} \beta_{n}-\bar{F}_{\alpha, \beta} \beta\right) \times_{\alpha, \beta} \phi_{n} \rightarrow 0 \quad \text { as } n \rightarrow \infty .
$$

Hence, $\bar{F}_{\alpha, \beta} \beta_{n} \stackrel{\Delta}{\rightarrow} \bar{F}_{\alpha, \beta} \beta$ as $n \rightarrow \infty$.

Finally, let $g_{n} \stackrel{\Delta}{\rightarrow} g$ in $B\left(\bar{L}_{\alpha, \beta}^{1}, \bar{D}, \times_{\alpha, \beta}, \bar{\Delta}\right)$ as $n \rightarrow \infty$. Then we find $J_{\alpha, \beta}^{d} \psi_{k} \in \bar{L}_{\alpha, \beta}^{1}$ such that $\left(g_{n}-g\right) \times_{\alpha, \beta} \phi_{k}=\left[\frac{J_{\alpha, \beta}^{d} \psi_{k} \times_{\alpha, \beta} \phi_{k}}{\phi_{k}}\right]$ and $J_{\alpha, \beta}^{d} \psi_{k} \rightarrow 0$ as $n \rightarrow \infty$ for some $\phi_{k}=J_{\alpha, \beta}^{d} \theta_{k}, \theta_{k} \in \Delta$. Now, using Definition 11, we obtain

$$
\left(\bar{F}_{\alpha, \beta}\right)^{-1}\left(\left(g_{n}-g\right) \times_{\alpha, \beta} \phi_{k}\right)=\left[\left(J_{\alpha, \beta}^{d}\right)^{-1} \frac{\left(J_{\alpha, \beta}^{d} \psi_{k} \times_{\alpha, \beta} J_{\alpha, \beta}^{d} \theta_{k}\right)}{J_{\alpha, \beta}^{d} \theta_{k}}\right] .
$$

That is,

$$
\left(\bar{F}_{\alpha, \beta}\right)^{-1}\left(\left(g_{n}-g\right) \times_{\alpha, \beta} \phi_{k}\right)=\left[\frac{\psi_{k} *_{\alpha, \beta} \theta_{k}}{\theta_{k}}\right]=\psi_{k} \rightarrow 0 \quad \text { as } k \rightarrow \infty \text { in } L_{\alpha, \beta}^{1} .
$$

Thus, Eq. (23) gives

$$
\left(\bar{F}_{\alpha, \beta}\right)^{-1}\left(\left(g_{n}-g\right) \times_{\alpha, \beta} \phi_{k}\right)=\left(\left(\bar{F}_{\alpha, \beta}\right)^{-1} g_{n}-\left(\bar{F}_{\alpha, \beta}\right)^{-1} g\right) *_{\alpha, \beta} \theta_{k} \rightarrow 0 \quad \text { as } n \rightarrow \infty .
$$


Due to the above equation, we infer that $\left(\bar{F}_{\alpha, \beta}\right)^{-1} g_{n} \stackrel{\Delta}{\rightarrow}\left(\bar{F}_{\alpha, \beta}\right)^{-1} g$ for large values of $n$ in $B\left(L_{\alpha, \beta}^{1}, D, *_{\alpha, \beta}, \Delta\right)$.

This ends the proof of the theorem.

Theorem 14 The extended $\bar{F}_{\alpha, \beta}$ transform and the classical $J_{\alpha, \beta}^{d}$ transform are consistent.

Proof For every $\psi \in L_{\alpha, \beta}^{1}$, assume that $\beta$ is its representative in $B\left(L_{\alpha, \beta}^{1}, D, *_{\alpha, \beta}, \Delta\right)$. This indeed reveals that $\beta=\left[\frac{\psi *_{\alpha}, \beta \delta_{n}}{\delta_{n}}\right]$, where $\left(\delta_{n}\right) \in \Delta, n \in \mathbb{N}$. It is obvious that the delta sequence $\left(\delta_{n}\right)$ is independent from the representative for all $n \in \mathbb{N}$. Consequently,

$$
\bar{F}_{\alpha, \beta}(\beta)=\bar{F}_{\alpha, \beta}\left(\left[\frac{\psi *_{\alpha, \beta} \delta_{n}}{\delta_{n}}\right]\right)=\left[\frac{J_{\alpha, \beta}^{d}\left(\psi *_{\alpha, \beta} \delta_{n}\right)}{J_{\alpha, \beta}^{d} \delta_{n}}\right]=\left[\frac{J_{\alpha, \beta}^{d} \psi \times_{\alpha, \beta} J_{\alpha, \beta}^{d} \delta_{n}}{J_{\alpha, \beta}^{d} \delta_{n}}\right],
$$

which is the representative of $J_{\alpha, \beta}^{d} \psi$ in $\bar{L}_{\alpha, \beta}^{1}$.

Hence, the proof of the theorem is ended.

Theorem $15 \operatorname{Let}\left[\frac{\psi_{n}}{\delta_{n}}\right] \in B\left(\bar{L}_{\alpha, \beta}^{1}, \bar{D}, \times_{\alpha, \beta}, \bar{\Delta}\right)$. Then the condition for $\left[\frac{\psi_{n}}{\delta_{n}}\right]$, which is necessary and sufficient, to be in the range of $\bar{F}_{\alpha, \beta}$ is that $\psi_{n}$ is in the range of $J_{\alpha, \beta}^{d}$ for every $n \in \mathbb{N}$.

Proof If $\left[\frac{\psi_{n}}{\delta_{n}}\right]$ is in the range of $\bar{F}_{\alpha, \beta}$, then indeed $\psi_{n}$ is in the range of $J_{\alpha, \beta}^{d}$ for all $n \in \mathbb{N}$. For the converse, if $\psi_{n}$ is in the range of $J_{\alpha, \beta}^{d}$ for all $n \in \mathbb{N}$, then we can find $f_{n} \in L_{\alpha, \beta}^{1}$ so that $J_{\alpha, \beta}^{d} f_{n}=\psi_{n}$ for all $n \in \mathbb{N}$. Since $\left[\frac{\psi_{n}}{\delta_{n}}\right] \in B\left(\bar{L}_{\alpha, \beta}^{1}, \bar{D}, \times_{\alpha, \beta}, \bar{\Delta}\right)$,

$$
\psi_{n} \times_{\alpha, \beta} \delta_{m}=\psi_{m} \times_{\alpha, \beta} \delta_{n} \quad \text { for all } m, n \in \mathbb{N} \text {. }
$$

Therefore, for some $f_{n} \in L_{\alpha, \beta}^{1}$ and $\varphi_{n} \in \Delta$, we find

$$
J_{\alpha, \beta}^{d}\left(f_{n} *_{\alpha, \beta} \varphi_{n}\right)=J_{\alpha, \beta}^{d}\left(f_{m} *_{\alpha, \beta} \varphi_{n}\right) \quad \text { for all } m, n \in \mathbb{N} \text {. }
$$

The fact that $J_{\alpha, \beta}^{d}$ is injective, implies that $f_{n} *_{\alpha, \beta} \varphi_{m}=f_{m} *_{\alpha, \beta} \varphi_{n}, m, n \in \mathbb{N}$.

Thus, $\frac{f_{n}}{\varphi_{n}}$ is a quotient of the sequences in $B\left(L_{\alpha, \beta}^{1}, D, *_{\alpha, \beta}, \Delta\right)$. Hence,

$$
\bar{F}_{\alpha, \beta}\left[\frac{f_{n}}{\varphi_{n}}\right]=\left[\frac{\psi_{n}}{\psi_{n}}\right] \text { for some }\left[\frac{f_{n}}{\varphi_{n}}\right] \in B\left(L_{\alpha, \beta}^{1}, D, *_{\alpha, \beta}, \Delta\right)
$$

Hence the theorem is proved.

\section{Conclusion}

The classical theory of the Jacobi-Dunkl integral operator of [1] is extended to a class of Boehmians. Every element of the classical space $L_{\alpha, \beta}^{1}$ is identified as a member of the Boehmian space $B\left(L_{\alpha, \beta}^{1}, D, *_{\alpha, \beta}, \Delta\right)$. Various embeddings and characteristics of the extended integral operator including an inversion formula are given in a generalized sense. Convergence with respect to $\delta$ and $\Delta$ is also discussed.

Acknowledgements

The authors would like to thank the reviewers for taking the time to read and improve the present article. 
Availability of data and materials

Please contact the authors for data requests.

\section{Competing interests}

The authors declare that they have no competing interests.

\section{Authors' contributions}

All authors contributed equally, read and approved the final manuscript.

\section{Author details}

${ }^{1}$ Department of Physics and Basic Sciences, Faculty of Engineering Technology, Al-Balqa Applied University, 11134, Amman, Jordan. ${ }^{2}$ Department of Mathematics, College of Sciences, Jazan University, Jazan, 45142, Saudi Arabia. ${ }^{3}$ Department of Mathematics, College of Arts and Sciences, Prince Sattam Bin Abdulaziz University, Wadi Aldawasir, Saudi Arabia.

\section{Publisher's Note}

Springer Nature remains neutral with regard to jurisdictional claims in published maps and institutional affiliations.

Received: 15 January 2021 Accepted: 19 July 2021 Published online: 31 July 2021

\section{References}

1. Ben Salem, N., Ahmed Salem, A.: Convolution structure associated with the Jacobi-Dunkl operator on $\mathbb{R}$. Ramanujan J. 12(3), 359-378 (2006)

2. Ben Salem, N., Samaali, T.: Hilbert transform and related topics associated with Jacobi-Dunkl operators of compact and noncompact types. Adv. Pure Appl. Math. 2, 367-388 (2011)

3. Srivastava, H.M., Luo, M., Raina, R.K.: A new integral transform and its applications. Acta Math. Sci. 35B(6), 1386-1400 (2015)

4. Chouchene, F.: Harmonic analysis associated with the Jacobi-Dunkl operator on ] - 2, 2[. J. Comput. Appl. Math. 178, 75-89 (2005)

5. Chouchane, F., Mili, M., Trimèche, K.: An Lp version of Hardy's theorem for the Jacobi-Dunkl transform. Integ. Trans. Spl. Funct. 15(3), 225-237 (2004)

6. Ben Mohamed, $\mathrm{H}$.: The Jacobi-Dunkl transform on $\mathbb{R}$ and the convolution product on new space of distributions. Ramanujan J. 21, 145-175 (2010)

7. Chouchane, F., Mili, M., Trimche, K.: Positivity of the intertwining operator and harmonic analysis associated with the Jacobi-Dunkl operator on $\mathbb{R}$. J. Anal. Appl. 1(4), 387-412 (2003)

8. Exton, H.: Certain hypergeometric functions of four variables. Bull. Soc. Math. Grece, (N.S.) 13, 104113 (1972)

9. El-Ouadiah, S., Daher, R.: Some results for the Jacobi-Dunkl transform in the space $L_{p}\left(\mathbb{R} ; A_{;}(x) d x\right)$. Casp. J. Math. Sci. 5(2), 98-105 (2016)

10. Erdelyi, A., Magnus, W., Oberhettinger, F., Tricomi, G.: Higher Transcendental Functions, vol. 2. MacGraw-Hill Book Company (1953)

11. Sharma, C., Parihar, C.L.: Hypergeometric functions of four variables (I). J. Indian Acad. Math. 11(2), 99115 (1989)

12. Bloom, W.R., Heyer, H.: Harmonic Analysis of Probability Measures on Hypergroups. de Gruyter, Berlin (1995)

13. Srivastava, H.M., Choi, J.: Zeta and q-Zeta Functions and Associated Series and Integrals. Elsevier, Amsterdam (2012)

14. Agarwal, P., Choi, J.: Certain fractional integral inequalities associated with pathway fractional integral operators. Bull. Korean Math. Soc. 53(1), 181-193 (2016)

15. Agarwal, P., Choi, J., Kachhia, K., Prajapati, J.C., Zhou, H.Z.: Some integral transforms and fractional integral formulas for the extended hypergeometric functions. Commun. Korean Math. Soc. 31(3), 591-601 (2016)

16. Al-Omari, S., Agarwal, P.: Some general properties of a fractional Sumudu transform in the class of Boehmians. Kuwait J. Sci. 43(2), 16-30 (2016)

17. Kaur, D., Agarwal, P., Rakshit, M., Chand, M.: Fractional calculus involving (p,q)-Mathieu type series. Appl. Math Nonlinear Sci. 5(2), 1-15 (2020)

18. Agarwal, P. A composition formula of the pathway integral transform operator. Note Mat. 34(2), 145-156 (2015)

19. Agarwal, P.: Certain integral transforms for the incomplete functions. Appl. Math. Inf. Sci. 9(4), 2161 (2015)

20. Exton, H.: Some integral representations and transformations of hypergeometric functions of four variables. Bull. Soc. Math. Grece, (N.S.) 14, 132140 (1973)

21. Nemzer, D.: Periodic Boehmians II. Bull. Aust. Math. Soc. 44, 271-278 (1991)

22. Al-Omari, S., Agarwal, P., Choi, J.: Real covering of the generalized Hankel-Clifford transform of Fox kernel type of a class of Boehmians. Bull. Korean Math. Soc. 52(5), 1607-1619 (2015)

23. Mikusiński, P.: Convergence of Boehmians. Jpn. J. Math. 9(1), 159-179 (1983)

24. Al-Omari, S., Jumah, G., Al-Omari, J., Saxena, D.: A new version of the generalized Kratzel-Fox integral operators. Mathematics 6(222), 1-12 (2018)

25. Mikusinski, P.: Boehmians and pseudoquotients. Appl. Math. Inf. Sci. 5, 192-204 (2011)

26. Al-Omari, S., Al-Omari, J.F.: Some extensions of a certain integral transform to a quotient space of generalized functions. Open Math. 13, 816-825 (2015)

27. Al-Omari, S., Baleanu, D.: The extension of a modified integral operator to a class of generalized functions. J. Comput. Anal. Appl. 24(2), 209-218 (2018)

28. Al-Omari, S.: Some remarks on short-time Fourier integral operators and classes of rapidly decaying functions. Math. Methods Appl. Sci. 41, 1-8 (2018)

29. Al-Omari, S.: On a class of generalized Meijer-Laplace transforms of Fox function type kernels and their extension to a class of Boehmians. Georgian Math. J. 24(1), 1-13 (2017)

30. Nemzer, D.: The Laplace transform on a class of Boehmians. Bull. Aust. Math. Soc. 46, 347-352 (1992) 
31. Al-Omari, S.: A study on a class of modified Bessel-type integrals in a Fréchet space of Boehmians. Bol. Soc. Parana. Mat. 38(4), 145-156 (2020)

32. Al-Omari, S., Araci, S., Al-Smadi, M., Gumah, G., Rabaieh, H.: Estimates of certain paraxial diffraction integral operator and its generalized properties. Adv. Differ. Equ. 403, 1-12 (2020)

33. Al-Omari, S.: Some estimate of a generalized Bessel-Struve transform on certain space of generalized functions. Ukr. Math. Bull. 69(9), 1155-1165 (2017)

34. Al-Omari, S., Kilicman, A.: An estimate of Sumudu transform for Boehmians. Adv. Differ. Equ. 2013, 77 (2013)

35. Al-Omari, S., Baleanu, D.: A quadratic-phase integral operator for sets of generalized integrable functions. Math. Methods Appl. Sci. 43(7), 4168-4176 (2020)

36. Karunakaran, V., Vembu, R.: Hillbert transform on periodic Boehmians. Houst. J. Math. 29, 439-454 (2005)

37. Al-Omari, S., Baleanu, D.: Some remarks on short-time Fourier integral operators and classes of rapidly decaying functions. Math. Methods Appl. Sci. 41(16), 5354-5361 (2019)

38. Al-Omari, S., Baleanu, D.: Quaternion Fourier integral operators for spaces of generalized quaternions. Math. Methods Appl. Sci. 41, 9477-9484 (2018)

39. Boehme, T.K.: The support of Mikusinski operators. Trans. Am. Math. Soc. 176, 319-334 (1973)

40. Fernandez, A., Baleanu, D.: Classes of operators in fractional calculus: a case study. Math. Methods Appl. Sci. (2021). To appear. https://doi.org/10.1002/mma.7341

41. Zemanian, A.H.: Generalized Integral Transformation. Dover, New York (1968) First Published by Interscience Publishers

42. Al-Omari, S.: Some characteristics of S transforms in a class of rapidly decreasing Boehmians. J. Pseudo-Differ. Oper. Appl. 5(4), 527-537 (2014)

43. Exton, H.: Multiple Hypergeometric Functions and Applications. Ellis Horwood, London (1976)

44. Al-Omari, S.: Natural transform in Boehmian spaces. Nonlinear Stud. 22(2), 291-297 (2015)

\section{Submit your manuscript to a SpringerOpen ${ }^{\circ}$ journal and benefit from:}

- Convenient online submission

- Rigorous peer review

- Open access: articles freely available online

- High visibility within the field

- Retaining the copyright to your article

Submit your next manuscript at $\gg$ springeropen.com 